\title{
Activation of selected trunk muscles during symmetric functional activities in poststroke hemiparetic and hemiplegic patients
}

\author{
Ruth Dickstein, Yael Heffes, Yocheved Laufer, Zvika Ben-Haim
}

\begin{abstract}
Objective-To compare the EMG activity between the recti abdominii muscles and between the lumbar erector spinae muscles in hemiparetic and hemiplegic patients during functional symmetric trunk movements and to compare patients' EMG activity profiles with those of healthy controls..

Methods-EMG activity from the selected muscles was recorded during three symmetric and time controlled trunk exercises. Data analysis was based on values of cross correlations and of ratios between EMG activity of the bilateral corresponding muscles.
\end{abstract}

Results-In all groups, the highest cross correlations were obtained for both muscles when the muscles acted as prime movers. For the recti abdominii muscles, these values in the patients were comparable with those of the healthy subjects, whereas for the extensor muscles, the highest synchronous activity was displayed in healthy subjects and the lowest in hemiplegic patients. Laterality differences in the amount of EMG activity of the recti abdominii muscles were not biased towards one side. For the extensor muscles, in the controls, the activation levels were higher in the left erector spinae muscle than in the right one in two of the three exercises. Similarly, in the extensor muscles of the hemiparetic patients, activity on the paretic side was higher than on the non-paretic side in two exercises.

Conclusions-In patients with a supratentorial poststroke hemiparesis or hemiplegia, bilateral corresponding axial trunk muscles co-contract during symmetric trunk activities. Synchronous activation is at its highest level during voluntary dynamic tasks and is greater in the recti abdominii than in the erector spinae muscles. For both muscles, EMG activation levels on the paretic side were not lower than on the non-paretic side. Thus, the assertion that the muscles on the paretic side are activated to a lesser extent than their counterparts on the non-paretic side during symmetric trunk movements was not confirmed.

(F Neurol Neurosurg Psychiatry 1999;66:218-221)

Keywords: stroke; hemiparesis; hemiplegia; rehabilitation; trunk

Hemiparesis caused by upper motor lesions is known to be more detrimental to distal than to proximal body musculature. ${ }^{1}$ This "proximal to distal" gradient of impairment is explained by the fact that motor neurons innervating axial and proximal limb muscles receive both ipsilateral and contralateral descending inputs, whereas peripheral muscles are supplied by motor neurons, of which the supraspinal input is mainly contralateral..$^{3-5}$ Hence, the rationale for the current emphasis placed by physical rehabilitation disciplines on restoration of the function of trunk and girdle muscles ${ }^{67}$ is perplexing. Furthermore, objective evidence of contralateral (opposite the lesion side) malfunction of axial and proximal muscles in poststroke hemiparetic patients is sparse, and what there is relates mainly to their role in postural adjustments $^{8}$ and not as prime movers. In the current work we questioned the assertion that axial muscles on the paretic side are less activated than their counterparts on the non-paretic side. We studied the function of the rectus abdominis and lumbar erector spinae muscles of hemiparetic and hemiplegic patients during basic symmetric functions of daily life.

\section{Methods}

SUBJECTS

The study involved 41 patients who were residents of a rehabilitation hospital and who had a single unilateral thromboembolic stroke in the territory of the anterior brain circulation and 20 healthy controls (eight men, mean age 69 (SD 7.5) years). The assignment of patients to either the "hemiparetic" or "hemiplegic" group was based on the severity of the paralysis as reflected by the ability to stand up without or with assistance respectively. There were 10 and 12 patients with left side paralysis and 11 and eight patients with right side paralysis (mean age 72.7 (SD 9) and 75.6 (SD 6.9) years) in the hemiparetic and hemiplegic group respectively. The hemiplegic patients scored substantially 

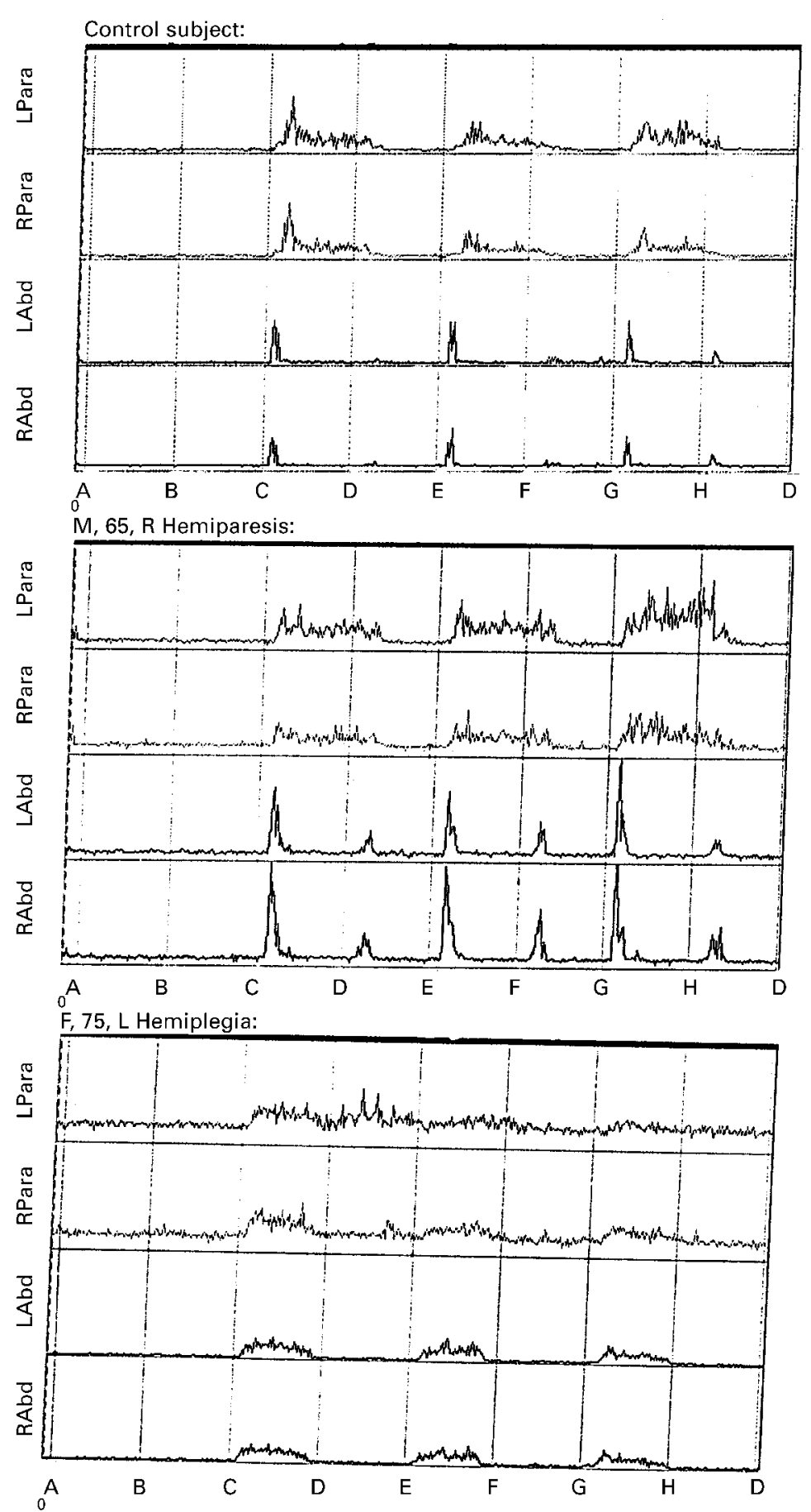

EMG activity profile of the left and right rectus abdominis (Labd and Rabd) and left and right lumbar erector spinae (Lpara and Rpara) muscles during trunk movements to an upright position from reclined sitting (exercise 1, three repetitions). Data shown are from one subject in each group. The time interval between each pair of vertical lines is 6 seconds. The interval between markers $A$ and $C$ designates the baseline period which is followed by the three repetitions of the exercise. lower than the hemiparetic patients on the Barthel index scale, ${ }^{9}$ the Brunnstrum scale, ${ }^{10}$ the trunk control test, ${ }^{11}$ and the functional reach test (performed while seated). ${ }^{12}$

INSTRUMENTATION AND PROTOCOL

The subjects were tested while seated on a chair (46 cm height) with side and vertical rests. Testing was initiated after skin preparation and attachment of EMG electrodes, and consisted of the following three exercises, each of which was performed three times.

(1) From reclined sitting $\left(120^{\circ}\right.$ hip flexion) and hands on contralateral shoulders, bringing the trunk forward beyond $90^{\circ}$ of hip flexion; (2) from upright sitting ( $90^{\circ}$ hip flexion) in a relaxed posture, shrugging shoulders backwards and extending the back; (3) from reclined sitting $\left(120^{\circ}\right.$ hip flexion) and hands clasped forward to standing up (the hemiplegic patients were unable to stand up unassisted and did not perform this exercise).

Electromyography data $(0-500 \mathrm{~Hz}$ bandwidth, $1000 \mathrm{~Hz}$ sampling frequency) were collected during a baseline period of 12 seconds as well as during the performance of three $6 \mathrm{sec}-$ ond long repetitions of each exercise. Interrepetition intervals were also 6 seconds long. The data were rectified and integrated over periods of $10 \mathrm{~ms}$ and stored for later analysis.

\section{DATA ANALYSIS}

Cross correlation was used to calculate the level of temporal synchronisation between the activity profile of corresponding bilateral muscles using a standard formula. ${ }^{13}$

The ratios between mean EMG activity of the same muscle on each side (left/right (L/R) in the controls and paretic/non-paretic (P/NP) in the patients) were also calculated and tested against the null hypothesis so that this ratio equals 1 .

One way analysis of variance (ANOVA) followed by post hoc Bonferroni pairwise comparisons of means was used for group comparisons. Student's $t$ tests were applied to compare the amount of activity between corresponding bilateral muscles.

\section{Results}

Examples of data recorded during the first exercise from one healthy subject, one hemiparetic patient, and one hemiplegic patient are depicted in the figure.

\section{TIMING OF MUSCULAR ACTIVATION}

Cross correlation values (table) between the two recti abdominii muscles were highest in

Mean values of cross correlation between left and right (paretic and non-paretic) lumbar erector spinae and between the left and right (paretic and non-paretic) rectus abdominis muscles in healthy controls and in hemiparetic and hemiplegic patients. Bringing trunk forward, extending back, standing up

\begin{tabular}{|c|c|c|c|c|c|c|}
\hline & Erec $s p$ & Rect $a b$ & Erec sp & Rect $a b$ & Erec sp & Rect $a b$ \\
\hline Controls & 0.71 & 0.84 & 0.69 & 0.54 & 0.82 & 0.63 \\
\hline Hemiplegic & 0.49 & 0.82 & 0.43 & 0.52 & & \\
\hline Hemiparetic & 0.65 & 0.81 & 0.73 & 0.55 & 0.73 & 0.58 \\
\hline ANOVA & $F=8.74, \mathrm{p}=0.0005$ & NS & $F=22.4, \mathrm{p}=0.0000$ & NS & $F=6.7, \mathrm{p}=0.01$ & \\
\hline Post hoc & $\star$ & & $\star$ & & $\star$ & \\
\hline
\end{tabular}

NS=Non-significant. ${ }^{\star}$ For exercises 1 and 2 implies a significant lower cross correlation value between the erector spinae (Erec sp) muscles in the hemiplegic patient group compared with the equivalent values in the other groups. For exercise 3 , the hemiparetic patients had significantly lower values than the controls. Rect $a b=$ recti abdominii. 
exercise, which involved concentric bilateral contraction. Between group differences, however, were not found in any of the three exercises. In the erector spinae evaluation, cross correlation between the two sides was significantly lower in the hemiplegic group compared with both the controls and the hemiparetic subjects in both exercise 1 and 2 . In exercise 3, cross correlation between the erector spinae of the two sides was significantly lower in the hemiparetic patients compared with the controls.

\section{AMOUNT OF MUSCULAR ACTIVATION}

For the controls, differences between the left and right erector spinae muscles were found in both exercise 1 and 3, with the left muscles being significantly more active than the right ones $(t=2.13, \mathrm{p}=0.03$ and $t=4, \mathrm{p}=0.0007$, respectively). No laterality differences were encountered for the rectus abdominis muscle in any of the three exercises. In the hemiparetic patients, the erector spinae muscles on the paretic side yielded a significantly higher activity level than those on the non-paretic side in exercises 2 and $3(t=2.18, \mathrm{p}=0.04$ and $t=2.05$, $\mathrm{p}=0.05$, respectively), whereas the rectus abdominis on the paretic side was less active in exercise $2(t=2.31, \mathrm{p}=0.03)$. In the hemiplegic group of patients, no laterality bias was found in both exercises and for both muscles.

\section{Discussion}

Synchronisation between EMG records of bilateral axial muscles is implied by high positive correlation values between the corresponding muscles and could indicate a common drive simultaneously subserving bilateral motor neuron pools. ${ }^{4}$ For the recti abdominii muscles, this degree of synchronisation was comparable in our three study groups, being the highest when the muscle served as a prime mover (exercise 1) and lower during stabilisation and restraint actions. These results are consistent with the findings of Carr et al for healthy subjects, ${ }^{5}$ and we further extended them to apply to poststroke patients. For the erector spinae muscles (exercises 2 and 3 ), the correlations between the two sides in the hemiplegic patients were significantly lower than in the healthy and hemiparetic subjects, indicating that for severely afflicted patients, simultaneous activity of both sides might be impaired both when the muscles serve as prime movers (exercise 2) or for restraint (exercise 1). Alternatively, comparable values of cross correlation were obtained for the healthy subjects and the hemiparetic patients, indicating that normal coactivation of corresponding bilateral muscles can prevail with slight paralysis. During the standing up action (exercise 3, the hemiplegic patients exempted), synchronous activity of the erector spinae muscles was the highest, pointing again to the association between high correlations and the role of the muscle as a prime mover. This correlation was higher in the healthy subjects than in the hemiparetic patients, implying that coactivation of the lumbar erector spinae muscles in hemiparetic patients could have deteriorated somewhat.
No laterality bias was found in any group in the amount of EMG activity of the recti abdominii muscles in the exercises which involved dynamic shortening of these muscles (exercises 1 and 3). The higher activation levels on the non-paretic side in the hemiparetic patients in exercise 2 do not seem to convey important information because the prime movers for performing this exercise are the erector spinae muscles, whereas the abdominal muscles display mainly low level tonic activity. In view of the comparability in the bilateral function of these muscles in both groups of patients and healthy controls, the claim of an existing malfunction of the abdominal muscles in poststroke patients ${ }^{6}$ was not confirmed. Moreover, the fact that the recti abdominii muscles displayed a normal coactivation pattern in the two patient groups provided compelling evidence of their intactness during performance of symmetric voluntary motor tasks. As for the erector spinae muscles, laterality differences in the magnitude of EMG activity were found both in the healthy subjects and in the hemiparetic patients in two of the three exercises: in the control group, the muscle on the left side had higher activity levels than the corresponding right one, whereas in the hemiparetic patients, the muscle on the paretic side showed higher activity levels than the corresponding muscle on the non-paretic side. A likely explanation for these findings might be related to the higher recruitment rate of motor neurons as an a priori compensation for real or perceived weakness. ${ }^{14}$ Such weakness, if it exists, would probably have been more apparent with more strenuous or longer lasting exercises. Noteworthy of mention here is the report by Gandevia and McCloskey, who found that hemiparetic patients consistently overestimate the magnitude of forces generated on the paretic side. ${ }^{15}$

Impairment of trunk control in hemiplegic or paretic patients has often been documented and characterised by asymmetry in performance of rotatory and side bending activities. ${ }^{11} 1617$ Trunk control has been shown to be a valid predictor of stroke rehabilitation outcome and to correlate positively with established functional and motor assessments. ${ }^{11}$ Nevertheless, the link between deficiencies in performance of trunk movements and malfunction of either individual or groups of trunk muscles has not been established. The findings of the current study pointed to normal function of the abdominal axial muscles in symmetric activities, whereas the results for the lumbar extensor muscles were less conclusive. Further clarification of the role of trunk muscles and of the nature of their impairment in poststroke patients is required in a broad repertoire of activities. The knowledge to be gained is imperative for planning rational rehabilitation strategies.

1 Colebatch JG, Gandevia SC. The distribution of muscular weakness in upper motor neuron lesions affecting the arm. Brain 1989;112:749-63.

2 Adams RW, Gandevia SC, Skuse NF. The distribution of muscle weakness in upper motor neuron lesions affecting the lower limb. Brain 1990;113:1459-76 
3 Brinkman J, Kuypers HGJM. Split brain monkeys: cerebral control of ipsilateral arm, hand and finger movements. Scicontrol of ipsilateral a
ence 1972;176:536-9

4 Kuypers HGJM. The anatomical organization of the descending pathways and their contributions to motor control especially in primates. In: J E Desmedt, ed. New developments in electromyography and clinical neurophysiology. Vol 3. Basel: Karger, 1973:38-68.

5 Carr LJ, Harrison LM, Stephens JA. Evidence for bilateral innervation of certain homologous motoneurone pools in man. F Physiol 1994;475:217-27.

6 Davies PM. Right in the middle - selective trunk activity in the treatment of adult hemiplegia. Berlin: Springer-Verlag, 1990. Bobath B. Adult hemiplegia: evaluation and treatment. 3rd ed. London: Butterworth Heinemann, 1991.

8 Palmer E, Downes L, Ashby P. Associated postural adjustments are impaired by lesions of the cortex. Neurology 1996;46:471-5.

9 Collin C, Wade DT, Davies S, et al. The Barthel ADL index: a reliability study. International Disability Studies 1988;10: 61-3.
10 Brunnstrum S. Movement therapy in hemiplegia, a neurophysiological approach. 1st ed. New York: Harper and Row, 1970

1 Collin C, Wade D. Assessing motor impairment after stroke: a pilot reliability study. F Neurol Neurosurg Psychiatry 1990 53:576-9.

12 Duncan PW, Studenski ST, Chandler J, et al. Functional reach: predictive validity in a sample of elderly male veterans. $\mathcal{F}$ Gerontol 1992;47:93-8.

13 De Luca CJ. Surface electromyography: what's new? Torino, taly: Neuromuscular Research Center, Boston University, CLUT, 1992

14 Bigland -Ritchie B. EMG/force relations and fatigue of human voluntary contractions. Exerc Sport Sci Rev 1981;9: 75-117.

15 Gandevia SC, McCloskey DI. Sensations of heaviness. Brain 1977;100:345-54

16 Franchignoni FP, Tesio L, Ricupero C, et al. Trunk control test as early predictor of stroke rehabilitation outcome. test as early predictor
Stroke $1997 ; 28: 1382-5$.

17 Bohannon RC. Recovery and correlates of trunk muscle strength after stroke. Int J Rehabil Res 1995;18:262-7. 